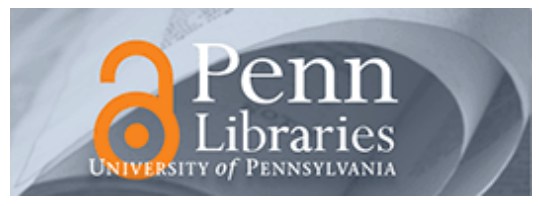

University of Pennsylvania

ScholarlyCommons

$5-1-1971$

\title{
Longitudinal Dynamical Susceptibility of the Heisenberg Ferromagnet at Short Wavelengths and Low Temperatures
}

\author{
A. Brooks Harris \\ University of Pennsylvania, harris@sas.upenn.edu
}

Follow this and additional works at: https://repository.upenn.edu/physics_papers

Part of the Quantum Physics Commons

\section{Recommended Citation}

Harris, A. (1971). Longitudinal Dynamical Susceptibility of the Heisenberg Ferromagnet at Short Wavelengths and Low Temperatures. Physical Review B, 3 (9), 3065-3071. http://dx.doi.org/10.1103/ PhysRevB.3.3065

This paper is posted at ScholarlyCommons. https://repository.upenn.edu/physics_papers/418

For more information, please contact repository@pobox.upenn.edu. 


\title{
Longitudinal Dynamical Susceptibility of the Heisenberg Ferromagnet at Short Wavelengths and Low Temperatures
}

\author{
Abstract \\ The longitudinal dynamical susceptibility of the Heisenberg ferromagnet is studied at short wavelengths \\ and low temperatures. It is shown that identical results to order $1 / \mathrm{S}$ are obtained using (a) a spin \\ decoupling technique, (b) a diagrammatic method using the Holstein-Primakoff transformation, and (c) a \\ diagrammatic method using the Dyson-Maleev transformation. We thus conclude that there are no \\ significant kinematic effects at low temperatures. Using the random-phase approximation, we find that \\ the Dyson-Maleev interactions between magnons are too weak to support the existence of a zero-sound \\ mode. Both these conclusions disagree with the recent results of other authors.

\section{Disciplines} \\ Physics | Quantum Physics
}


${ }^{27}$ A. J. Heeger, in Ref. 26.

${ }^{28}$ A. A. Abrikosov, Physics (N. Y.) 2, 61 (1965).

${ }^{29}$ G. G. Low and T. M. Holden, Proc. Phys. Soc. (London) 89, 119 (1966).

${ }^{30} \mathrm{An}$ analysis of the detailed magnetoresistance of the Pd 0.1-at. \% Co alloy over a wide range of magnetic fields and temperatures (which will be the subject of a further publication) yields values of $g=2.9 \pm 0.1$ and $S=4.7 \mp 0.2$. Using this value of $S$ in the present analysis yields $J_{\text {eff }}(5 \mathrm{~K})=0.053 \mathrm{eV}, n_{s}\left(E_{F}\right)=2.5 / \mathrm{eV}$ atom, and $J=0.14 \mathrm{eV}$.

${ }^{31} \mathrm{C}$. Herring, in Magnetism, edited by G. T. Rado and H. Suhl (Academic, New York, 1966), Vol. IV.

${ }^{32} \mathrm{~J}$. R. Schrieffer and P. A. Wolff, Phys. Rev. 149 , 491 (1966).

${ }^{33}$ In the Anderson model [P. W. Anderson, Phys. Rev. 124,41 (1961)], the total polarization has two contributions, a positive polarization resulting from the admixture effect (which can be exchange enhanced to give a. "giant" moment) and a negative contribution from the energy-shift term. While the total polarization may be positive under certain circumstances (in exchange-enhanced matrices), the Kondo effect results from the energy-shift term alone, and the relevent exchange interaction from this source is always antiferromagnetic.

${ }^{34}$ K. Fischer, Phys. Rev. 158, 618 (1967).

${ }^{35}$ A. Blandin, J. Appl. Phys. 39 , 1285 (1968).

${ }^{36}$ G. S. Knapp, Phys. Letters $25 \mathrm{~A}, 114$ (1967).

${ }^{37}$ The decrease in $\Delta \rho_{\text {spin }}$ is approximately $40 \%$ over this temperature range if $S$ is taken to be 4.7 , as determined from magnetoresistance measurements. This would still suggust a Kondo temperature below $1 \mathrm{~K}$.

${ }^{38}$ P. Lederer and D. L. Mills, Phys. Rev. 165, 837 (1968).

${ }^{39}$ A. B. Kaiser and S. Doniach, Intern. J. Magnetism 1, $11(1970)$.

${ }^{40}$ M. Levine and H. Suhl, Phys. Rev. 171,567 (1968).

${ }^{41}$ D. R. Hamann, Phys. Rev. Letters $\underline{23}, 95$ (1969).

\title{
Longitudinal Dynamical Susceptibility of the Heisenberg Ferromagnet at Short Wavelengths and Low Temperatures*
}

\author{
A. Brooks Harris \\ Department of Physics, University of Pennsylvania, Philadelphia, Pennsylvania 19104
}

(Received 13 January 1971)

\begin{abstract}
The longitudinal dynamical susceptibility of the Heisenberg ferromagnet is studied at short wavelengths and low temperatures. It is shown that identical results to order $1 / S$ are obtained using (a) a spin decoupling technique, (b) a diagrammatic method using the Holstein-Primakoff transformation, and (c) a diagrammatic method using the Dyson-Maleev transformation. We thus conclude that there are no significant kinematic effects at low temperatures. Using the random-phase approximation, we find that the Dyson-Maleev interactions between magnons are too weak to support the existence of a zero-sound mode. Both these conclusions disagree with the recent results of other authors.
\end{abstract}

\section{INTRODUCTION}

The increasing resolution attainable in inelastic neutron-scattering experiments has stimulated interest in the various collective excitations in magnetic systems, since these excitations are potentially observable via such experiments. Thus, detailed investigations of two-spin-wave bound states, ${ }^{1-4}$ of second magnons, ${ }^{5-7}$ and more recently, of zero sound ${ }^{8,9}$ have been carried out. With regard to zero sound, the work of Ranninger and Natoli $(\mathrm{RN})^{9}$ is especially provocative. By analyzing the longitudinal dynamical susceptibility $\mathrm{RN}$ have concluded that (a) there is a well-defined collective excitation for wave vectors near the zone boundary, and (b) kinematic interactions play an important role in the kinematics of this mode. The purpose of this paper is to investigate these points in greater detail, since such conclusions have rather fundamental implications for both theoretical and experimental programs in magnetism.

The motivation for reexamining these conclusions is that the theory of $\mathrm{RN}$ appears to embody two physically unsatisfactory aspects. First, they claim to have detected effects of the kinematic interaction on the zero-sound mode, but the effects they find are simply proportional to various powers of Bose occupation numbers. In other words, the kinematic interaction in their theory gives rise to effects of $\operatorname{order}(k T / J S)^{n}$, where $n$ is of order 3 . On the other hand, such large effects at low temperature are not to be expected in view of Dyson's arguments, ${ }^{10}$ which suggest that these effects are of order $\xi \equiv e^{-a T_{C} / T}$, where $T_{C}$ is the Curie temperature and $a$ is a constant of order unity. Indeed, up to now, no one has been able to construct a theory which is accurate enough to detect effects of order $\xi$ at low temperatures. (In this connection, it is worth noting that treatments of the two-spin-wave bound states via a hard-core potential, ${ }^{11,12}$ which rigorously exclude kinematic effects, have thus far only taken account of two-spin-wave states, and hence do not yield any conclusions about the kinematic effects of states involving more than two spin 
waves. ) The second unsatisfactory feature of the theory of RN concerns their treatment of the interaction between the zone-boundary magnon and the long-wavelength acoustic magnon which are bound together to form a zero-sound excitation. In theory, the best way to describe this interaction is to use the two-spin-wave $t$ matrix ${ }^{1-3,13}$ which properly includes the effect of repeated scatterings between spin waves. Unfortunately, such a treatment becomes excessively complicated, because this complicated interaction is part of the kernel of an integral equation whose solution yields the zero-sound mode. A simple, but physically correct approximation may be obtained using the Dyson-Maleev (DM) interaction ${ }^{10,14}$ to describe the scattering between spin waves. In this context, the HolsteinPrimakoff (HP) $)^{15}$ interaction is not suitable because it leads to large interactions between long-wavelength spin waves. Of course, if the full $t$ matrix were used to describe spin-wave interactions, then both formalisms would no doubt give the correct weak interaction between a long-wavelength spin wave and the zone-boundary magnon. ${ }^{16}$ Similarly, it can be seen that the interaction implied by the decoupling scheme of $\mathrm{RN}$, like the HP interaction, is too strong, since it does not vanish when one of the interacting magnons has an infinitely long wavelength. The same comments can be made about the results of $\mathrm{Liu}^{17}$ and of Reiter ${ }^{7}$ in the collisionless regime. Using the physically correct weaker interaction between spin waves, we find that the zerosound mode is not well defined at low temperatures. At higher temperatures, where the DM formalism becomes inappropriate, it is possible, although unlikely, that such a collective excitation does exist.

Briefly, this paper is organized as follows. In Sec. II we show that to lowest nontrivial order in $1 / S$ the same results are obtained for the longitudinal dynamical susceptibility using (a) the decoupling technique of RN (modified to include correctly spin kinematics), (b) the diagrammatic formalism of $\mathrm{RN}$ using the HP transformation to bosons, and (c) the diagrammatic formalism of RN using the DM transformation. On the basis of this calculation, we conclude that the kinematic effects found by $\mathrm{RN}$ are spurious. In Sec. III we use the DM transformation to study the possible existence of a zerosound mode at short wavelengths. As mentioned above, the interactions between spin waves in the DM picture are too weak to support a well-defined zero-sound mode. A brief summary of our work is given in Sec. IV. The correlation function $\left\langle S_{\overrightarrow{\mathrm{k}}} S_{-\mathrm{k}}\right\rangle$ is evaluated in the Appendix.

\section{EQUIVALENCE OF THE VARIOUS METHODS TO ORDER $1 / S$}

We treat the usual model of a Heisenberg ferromagnet with nearest-neighbor interactions on a simple cubic lattice:

$$
\mathfrak{H}=-J \sum_{\vec{R}, \vec{b}} \vec{S}_{\mathbb{R}} \cdot \vec{S}_{\vec{R}+\vec{b}},
$$

where the sum over $\vec{\delta}$ is carried over the six nearest-neighbor vectors. In terms of the Fourier transformed variables, the Hamiltonian may be written as

$$
\mathfrak{H}=-J_{z} \sum_{\overrightarrow{\mathbf{k}}} \gamma_{\overrightarrow{\mathbf{R}}} \overrightarrow{\mathrm{S}}_{\overrightarrow{\mathbf{k}}} \cdot \overrightarrow{\mathrm{S}}_{-\overrightarrow{\mathbf{k}}},
$$

where

$$
\begin{aligned}
& \vec{S}_{\mathbf{k}}=N^{-1 / 2} \sum_{\overrightarrow{\mathrm{R}}} \vec{S}_{\vec{R}} e^{i \overrightarrow{\mathrm{k}} \cdot \overrightarrow{\mathrm{R}}}, \\
& \gamma_{\overrightarrow{\mathrm{k}}}=z^{-1} \sum_{\vec{b}} e^{i \overrightarrow{\mathrm{k}} \cdot \vec{b}},
\end{aligned}
$$

where $z$ is the number of nearest neighbors, $z=6$. (Note that the usual definition of $\gamma_{\vec{k}}$ which we use here differs by a factor of $z$ from that of RN. )

To study the longitudinal susceptibility, one naturally wishes to express $S_{z}$ in terms of the transverse spin components, since these approximate the true normal-mode operators. Thus we write

$S_{\vec{R}_{z}}=S-\frac{1}{2 S} S_{\vec{R}}^{-} S_{\mathrm{R}}^{+}-\frac{1}{4 S^{2}} \frac{1}{(2 S-1)} S_{\bar{R}} S_{\vec{R}}^{-} S_{\mathrm{R}}^{+} S_{\mathrm{R}}^{+} \cdots$.

For the static properties, Oguchi ${ }^{16}$ has shown that the DM and the HP formalisms agree, if terms in perturbation theory are grouped according to the parameter $1 / S$. We shall apply this idea to the longitudinal dynamical susceptibility, and therefore we treat $1 / S$ as a small parameter. (The correct method of calculation for spin- $\frac{1}{2}$, for instance, is less clear cut, although, as we shall see, there are indications that the DM formalism is the best one to use in that case.) For large $S$ we write Eq. (5a) as

$$
\begin{aligned}
& S_{\overrightarrow{\mathrm{R}}_{Z}}=S-(2 S)^{-1} S_{\mathrm{R}} S_{\mathrm{R}}^{+}-(2 S)^{-3} S_{\mathrm{R}} S_{\mathrm{R}} S_{\mathrm{R}}^{+} S_{\mathrm{R}}^{+} \cdots, \\
& S_{\overrightarrow{\mathrm{k}} z}=N^{1 / 2} S \delta_{\overrightarrow{\mathrm{k}}, 0}-\left(\frac{1}{2 N^{1 / 2} S}\right) \sum_{\overrightarrow{\mathrm{k}}^{\prime}} S_{\mathrm{R}^{\prime}}^{-} S_{\mathrm{k}^{\mathrm{k}}-\overrightarrow{\mathrm{k}}^{\prime}}^{+}
\end{aligned}
$$

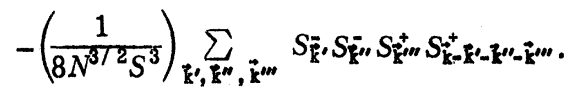

Since the longitudinal susceptibility is given as ${ }^{18}$

$$
\chi_{\bar{q}}^{z z}(t)=\left\langle\left\langle S_{\bar{q}}^{z} ; S_{-\bar{q}}^{z}\right\rangle\right\rangle \equiv-i \theta(t)\left\langle\left[S_{\bar{q}}^{z}(t), S_{-\bar{q}}^{z}(0)\right]\right\rangle,
$$

in the usual notation, we may write

$$
\begin{aligned}
& \chi_{\overrightarrow{\mathrm{a}}}^{z z}(t)=-\sum_{\overrightarrow{\mathrm{k}}}\left\langle\left\langle S_{\overrightarrow{\mathrm{k}}}^{-} S_{\overrightarrow{\mathrm{d}}-\overrightarrow{\mathrm{k}}}^{+}+\left(4 N S^{2}\right)^{-1}\right.\right. \\
& \left.\left.\times \sum_{\overrightarrow{\mathrm{k}}^{\prime}, \overrightarrow{\mathrm{k}}^{\prime \prime}} S_{\overrightarrow{\mathbf{k}}-\overrightarrow{\mathrm{k}}^{\prime}-\overrightarrow{\mathrm{k}}^{\prime \prime}}^{-} S_{\overrightarrow{\mathrm{k}}^{\prime}}^{+} S_{\overrightarrow{\mathrm{k}}^{\prime \prime}}^{+} S_{\overrightarrow{\mathrm{q}}-\overrightarrow{\mathrm{k}}}^{+} ; S_{-\overrightarrow{\mathrm{l}}}^{2}\right)\right\rangle / 2 S N^{1 / 2} .
\end{aligned}
$$

Since interactions between spin waves are of order $1 / S$, it is clear that to order $1 / S$ we only need to take account of free propagation in the higher-order 
Green's functions. Thus we have

$$
\begin{aligned}
\chi_{\overrightarrow{\mathbf{q}}}^{z z}(t) \approx & -\sum_{\overrightarrow{\mathbf{k}}}\left\langle\left\langle S_{\overrightarrow{\mathbf{k}}}^{+} S_{\overrightarrow{\mathbf{q}}-\overrightarrow{\mathrm{k}}}^{+} ; S_{-\mathbf{q}}^{z}\right\rangle\right\rangle \\
& \times\left(1+N^{-1} S^{-2} \sum_{\overrightarrow{\mathbf{k}}^{\prime}}\left\langle S_{\overrightarrow{\mathbf{k}}^{\prime}}^{-} S_{-\overrightarrow{\mathbf{k}}^{-}}^{+}\right\rangle\right) /\left(2 S N^{1 / 2}\right) \\
\approx & -\sum_{\overrightarrow{\mathbf{k}}}\left\langle\left\langle S_{\overrightarrow{\mathbf{k}}^{-}} S_{\mathbf{q}_{-}-\overrightarrow{\mathbf{k}}}^{+} ; S_{-\overrightarrow{\mathbf{q}}}^{z}\right\rangle\right\rangle[1+(2 n / S)] /\left(2 S N^{1 / 2}\right),
\end{aligned}
$$

where $n=N^{-1} \sum_{\overrightarrow{\mathbf{k}}} n_{\overrightarrow{\mathbf{k}}}$ and $n_{\overrightarrow{\mathbf{k}}}=\left[\exp \left(\hbar \omega_{\overrightarrow{\mathbf{k}}} / k T\right)-1\right]^{-1}$, where $\hbar \omega_{\overrightarrow{\mathbf{k}}}$ is the spin-wave energy and $T$ is the temperature. The evaluation of $\left\langle S_{\overrightarrow{\mathbf{k}}^{\prime}}^{-} S_{-\overrightarrow{\mathrm{k}}^{\prime}}^{+}\right\rangle$we have used in Eq. (9b) is correct for large $S$. For general $S$ we have evaluated this quantity in the Appendix.

It is clear that we need to determine $G(\vec{k}, \vec{q} ; \omega)$, which is defined as

$$
G(\overrightarrow{\mathrm{k}}, \overrightarrow{\mathrm{q}} ; \omega) \equiv N^{-1 / 2}\left\langle\left\langle S_{-\overrightarrow{\mathrm{k}}}^{-} S_{\overrightarrow{\mathrm{k}}+\mathrm{d}}^{+} ; S_{-\mathrm{q}}^{z}\right\rangle\right\rangle_{\omega},
$$

where we denote the temporal Fourier transform of the Green's functions by the subscript $\omega$. We use the decoupling technique of $\mathrm{RN}$ to obtain the following equation for $G$ :

$$
\begin{aligned}
& \hbar\left(\omega-\omega_{\overrightarrow{\mathbf{z}}+\overrightarrow{\mathrm{q}}}+\omega_{\overrightarrow{\mathrm{k}}}\right) G(\overrightarrow{\mathrm{k}}, \overrightarrow{\mathrm{q}} ; \omega) \\
& =-(2 \pi N)^{-1}\left(\left\langle S_{\overrightarrow{\mathbf{k}}}^{-} S_{-\overrightarrow{\mathrm{k}}}^{+}\right\rangle-\left\langle S_{\overrightarrow{\mathbf{k}}+\vec{q}} S_{-\overrightarrow{\mathrm{k}}-\overrightarrow{\mathbf{q}}}^{+}\right\rangle\right) \\
& +(J z / N S) \sum_{\overrightarrow{\mathbf{k}}^{\prime}}\left[\left(\gamma_{\mathfrak{q}}+\gamma_{\overrightarrow{\mathbf{k}}-\overrightarrow{\mathbf{k}}^{\prime}}-\gamma_{\overrightarrow{\mathbf{k}}^{\prime}}-\gamma_{\overrightarrow{\mathbf{k}}+\overrightarrow{\mathbf{q}}}\right)\left\langle S_{\overrightarrow{\mathbf{k}}+\overrightarrow{\mathrm{q}}}^{-} S_{-\overrightarrow{\mathbf{k}^{-}}-\overrightarrow{\mathrm{q}}}^{+}\right\rangle\right. \\
& \left.-\left(\gamma_{\vec{q}}+\gamma_{\overrightarrow{\mathbf{k}}-\overrightarrow{\mathbf{k}}^{\prime}}-\gamma_{\overrightarrow{\mathbf{k}}}-\gamma_{\overrightarrow{\mathbf{k}}^{\prime}+\vec{q}}\right)\left\langle S_{\overrightarrow{\mathbf{k}}}^{-} S_{-\overrightarrow{\mathbf{k}}}^{+}\right\rangle\right] G\left(\overrightarrow{\mathbf{k}}^{\prime}, \overrightarrow{\mathrm{q}} ; \omega\right) \text {. }
\end{aligned}
$$

Note that we have not set $\left\langle S_{\vec{k}}^{-} S_{-\vec{k}}^{+}\right\rangle=2 S n_{\vec{k}}$ as RN did in their Eq. (11), and also we have corrected a sign error in the last term on the right-hand side of their equation. To obtain a correct result in the susceptibility to order $1 / S$, it is necessary to use (see the Appendix)

$$
\left\langle S_{\overrightarrow{\mathrm{k}}}^{-} S_{-\overrightarrow{\mathrm{k}}}^{+}\right\rangle=2(S-n) n_{\overrightarrow{\mathrm{k}}}
$$

in the discontinuity term in Eq. (11). Elsewhere the approximation $\left\langle S_{\overrightarrow{\mathbf{k}}}^{-} S_{-\overrightarrow{\mathrm{k}}}^{+}\right\rangle=2 S n_{\overrightarrow{\mathrm{k}}}$ will suffice. Thus we obtain

$$
\begin{aligned}
\hbar(\omega- & \left.\omega_{\overrightarrow{\mathbf{k}}+\vec{q}}+\omega_{\vec{k}}\right) G(\overrightarrow{\mathbf{k}}, \overrightarrow{\mathrm{q}} ; \omega) \\
= & -(S / \pi N)\left(n_{\overrightarrow{\mathbf{k}}}-n_{\overrightarrow{\mathbf{k}}+\vec{q}}\right)[1-(n / S)] \\
& +(2 J z / N) \sum_{\overrightarrow{\mathbf{k}^{\prime}}}\left[\left(\gamma_{\vec{q}}+\gamma_{\mathbf{k}_{-}-\overrightarrow{\mathbf{k}}^{\prime}}-\gamma_{\overrightarrow{\mathbf{k}}^{\prime}}-\gamma_{\overrightarrow{\mathbf{k}}+\vec{q}}\right) n_{\overrightarrow{\mathbf{k}}+\overrightarrow{\mathbf{q}}}\right. \\
& \left.-\left(\gamma_{\overrightarrow{\mathbf{q}}}+\gamma_{\overrightarrow{\mathbf{k}}-\overrightarrow{\mathbf{k}}^{\prime}}-\gamma_{\overrightarrow{\mathbf{k}}}-\gamma_{\overrightarrow{\mathbf{k}}^{\prime}+\vec{q}}\right) n_{\overrightarrow{\mathbf{k}}}\right] G\left(\overrightarrow{\mathrm{k}}^{\prime}, \overrightarrow{\mathrm{q}} ; \omega\right) .
\end{aligned}
$$

Solving this equation by iteration up to order $1 / S$ and inserting the result into Eq. (9b), we obtain

$$
\chi_{\vec{q}}^{z z}(\omega)=(2 \pi N)^{-1}\left(1+\frac{n}{s}\right) \sum_{\overrightarrow{\mathbf{k}}} \frac{n_{\overrightarrow{\mathbf{k}}}-n_{\overrightarrow{\mathbf{k}}^{\prime}+\vec{q}}}{\hbar\left(\omega-\omega_{\overrightarrow{\mathbf{k}}+\vec{q}}+\omega_{\overrightarrow{\mathbf{k}}}\right)}
$$

$$
\begin{aligned}
& -\left(2 \pi N^{2} S\right)^{-1} \sum_{\overrightarrow{\mathbf{k}}, \overrightarrow{\mathrm{k}}^{\prime}}\left(\frac{\left(n_{\mathbf{k}^{\prime}}-n_{\overrightarrow{\mathbf{k}}^{\prime}+\overrightarrow{\mathrm{q}}}\right)}{\hbar\left(\omega-\omega_{\overrightarrow{\mathbf{k}}^{\prime}+\overrightarrow{\mathrm{q}}^{\prime}}+\omega_{\overrightarrow{\mathrm{k}}^{\prime}}\right)\left(\omega-\omega_{\overrightarrow{\mathrm{k}}+\overrightarrow{\mathrm{q}}^{\prime}}+\omega_{\overrightarrow{\mathbf{k}}}\right)}\right. \\
& \times\left[n_{\overrightarrow{\mathbf{k}}}\left(\omega_{\overrightarrow{\mathbf{k}}}+\omega_{\overrightarrow{\mathbf{k}}^{\prime}+\overrightarrow{\mathrm{a}}}-\omega_{\overrightarrow{\mathbf{q}}}-\omega_{\overrightarrow{\mathbf{k}}-\overrightarrow{\mathbf{k}}^{\prime}}\right)\right. \\
& \left.\left.-n_{\overrightarrow{\mathbf{k}^{+}}+\vec{q}}\left(\omega_{\overrightarrow{\mathrm{k}}^{\prime}}+\omega_{\overrightarrow{\mathrm{k}}+\overrightarrow{\mathrm{q}}}-\omega_{\overrightarrow{\mathrm{q}}}-\omega_{\overrightarrow{\mathrm{k}}-\overrightarrow{\mathrm{k}}^{\prime}}\right)\right]\right)
\end{aligned}
$$

for the longitudinal dynamical susceptibility correct to order $1 / S$.

As $\mathrm{RN}$ have done, one can also study the same function in the HP formalism. To avoid confusion we denote Green's function analogous to $G$ by $H(\overrightarrow{\mathrm{k}}, \overrightarrow{\mathrm{q}} ; \omega)$. Apart from a factor $-1 / N$, our $H$ is identical to $G(\overrightarrow{\mathrm{k}}, \overrightarrow{\mathrm{q}} ; \omega)_{\mathrm{HP}}$ of $\mathrm{RN}$. Correcting some algebraic errors, we find that their Eq. (20) gives

$$
\begin{aligned}
\hbar\left(\omega-\omega_{\overrightarrow{\mathbf{k}}+\overrightarrow{\mathrm{q}}}\right. & \left.+\omega_{\overrightarrow{\mathbf{k}}}\right) H(\overrightarrow{\mathrm{k}}, \overrightarrow{\mathrm{q}} ; \omega)=(S / \pi)\left(n_{\overrightarrow{\mathbf{k}}}-n_{\overrightarrow{\mathbf{k}}+\overrightarrow{\mathrm{q}}}\right) \\
& +(J z / N) \sum_{\overrightarrow{\mathrm{k}}^{\prime}}\left(n_{\overrightarrow{\mathbf{k}}+\overrightarrow{\mathrm{q}}}-n_{\overrightarrow{\mathbf{k}}}\right)\left(2 \gamma_{\overrightarrow{\mathrm{q}}}+2 \gamma_{\overrightarrow{\mathrm{k}}-\overrightarrow{\mathrm{k}}^{\prime}}-\gamma_{\overrightarrow{\mathbf{k}}}-\gamma_{\overrightarrow{\mathrm{k}}^{\prime}}\right. \\
& \left.\quad-\gamma_{\overrightarrow{\mathbf{k}}+\overrightarrow{\mathrm{q}}}-\gamma_{\overrightarrow{\mathbf{k}}^{\prime}+\overrightarrow{\mathrm{q}}}\right) H\left(\overrightarrow{\mathrm{k}^{\prime}}, \overrightarrow{\mathrm{q}} ; \omega\right) .
\end{aligned}
$$

In the HP formalism one has

$$
\chi_{\vec{q}}^{z z}(\omega)=(2 S)^{-1} \sum_{\overrightarrow{\mathbf{k}}} H(\overrightarrow{\mathrm{k}}, \overrightarrow{\mathrm{q}} ; \omega),
$$

so that iteration of Eq. (15) yields

$$
\begin{aligned}
& \chi_{\overrightarrow{\mathbf{q}}}^{z z}(\omega)_{\mathrm{HP}}=(2 \pi N)^{-1} \sum_{\overrightarrow{\mathbf{k}}} \frac{n_{\overrightarrow{\mathrm{k}}}-n_{\overrightarrow{\mathbf{k}}+\overrightarrow{\mathrm{a}}}}{\hbar\left(\omega-\omega_{\overrightarrow{\mathbf{k}}+\overrightarrow{\mathrm{q}}}+\omega_{\overrightarrow{\mathrm{k}}}\right)}
\end{aligned}
$$

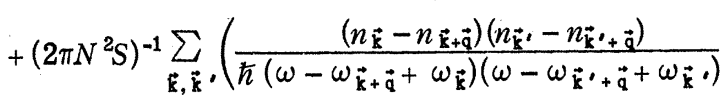

$$
\begin{aligned}
& \left.\times\left(\omega_{\overrightarrow{\mathbf{q}}}+\omega_{\overrightarrow{\mathbf{k}}-\overrightarrow{\mathbf{k}}},-\frac{1}{2} \omega_{\overrightarrow{\mathbf{k}}}-\frac{1}{2} \omega_{\overrightarrow{\mathbf{k}}},-\frac{1}{2} \omega_{\overrightarrow{\mathbf{k}}+\overrightarrow{\mathrm{q}}}-\frac{1}{2} \omega_{\overrightarrow{\mathbf{k}}}+\overrightarrow{\mathrm{q}}\right)\right)
\end{aligned}
$$

correct to order $1 / S$, where the subscripts HP indicate the result derived within the HolsteinPrimakoff formalism.

The results using the DM transformation can be readily inferred from the form of Eq. (15). All one must do is to replace the HP interaction $V_{\text {HP }}$ by the DM interaction $V_{\mathrm{DM}}$. From Eq. (15) we have

$V_{\mathrm{HP}}=(J z / 4 N)\left(2 \gamma_{\vec{q}}+2 \gamma_{\overrightarrow{\mathbf{k}}-\overrightarrow{\mathrm{k}}},-\gamma_{\overrightarrow{\mathbf{k}}}-\gamma_{\overrightarrow{\mathbf{k}}},-\gamma_{\overrightarrow{\mathrm{k}}+\overrightarrow{\mathrm{q}}}-\gamma_{\overrightarrow{\mathbf{k}}}{ }^{\prime}+\overrightarrow{\mathrm{q}}\right)$,

whereas the DM interaction is ${ }^{10,14}$

$$
V_{\mathrm{DM}}=(J z / 2 N)\left(\gamma_{\overrightarrow{\mathrm{q}}}+\gamma_{\overrightarrow{\mathbf{k}}-\overrightarrow{\mathrm{k}}},-\gamma_{\overrightarrow{\mathbf{k}}}-\gamma_{\overrightarrow{\mathbf{k}} \cdot+\overrightarrow{\mathrm{q}}}\right) \equiv V_{\overrightarrow{\mathbf{k}}, \overrightarrow{\mathbf{k}}} \cdot
$$

Using this interaction and repeating the steps leading to Eq. (17) we find that

$$
\begin{aligned}
& \chi_{\vec{q}}^{z z}(\omega)_{D M}=(2 \pi N)^{-1} \sum_{\overrightarrow{\mathbf{k}}} \frac{n_{\overrightarrow{\mathbf{k}}}-n_{\overrightarrow{\mathrm{k}}+\overrightarrow{\mathrm{q}}}}{\hbar\left(\omega-\omega_{\overrightarrow{\mathrm{k}}+\overrightarrow{\mathrm{q}}}+\omega_{\overrightarrow{\mathrm{k}}}\right)}
\end{aligned}
$$

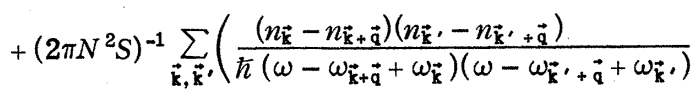




$$
\left.\times\left(\omega_{\vec{a}}+\omega_{\vec{k}-\vec{k}^{\prime}}-\omega_{\vec{k}}-\omega_{\vec{k}}+\vec{a}\right)\right)
$$

correct to order $1 / S$, where the subscripts DM indicate the result derived within the Dyson-Maleev formalism.
Let us now compare the results for the longitudinal susceptibility, Eqs. (14), (17), and (20). From the symmetry between $\vec{k}$ and $\vec{k}^{\prime}$ it is clear that Eqs. (17) and (20) are equivalent. Let us now show that Eqs. (14) and (17) give the same result. We have from Eqs. (14) and (17)

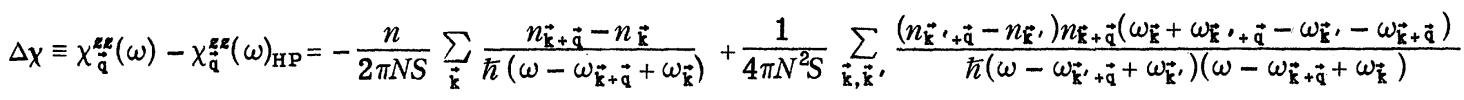

$$
\begin{aligned}
& +\frac{1}{4 \pi N^{2} S} \sum_{\overrightarrow{\mathbf{k}}, \overrightarrow{\mathbf{k}}^{\prime}} \frac{\left(n_{\overrightarrow{\mathbf{k}}^{\prime}}+\vec{q}-n_{\overrightarrow{\mathbf{k}}}\right) n_{\overrightarrow{\mathbf{k}}}\left(\omega_{\overrightarrow{\mathbf{k}}^{\prime}}+\omega_{\overrightarrow{\mathbf{k}}^{\prime}}+\vec{q}-\omega_{\overrightarrow{\mathbf{k}}},-\omega_{\overrightarrow{\mathbf{k}}+\vec{q}}\right)}{\hbar\left(\omega-\omega_{\overrightarrow{\mathbf{k}}^{\prime}+\vec{q}}+\omega_{\overrightarrow{\mathbf{k}}^{\prime}}\right)\left(\omega-\omega_{\overrightarrow{\mathbf{k}}+\vec{q}}+\omega_{\overrightarrow{\mathbf{k}}}\right)}
\end{aligned}
$$

Again, use of the symmetry between $\overrightarrow{\mathrm{k}}$ and $\overrightarrow{\mathrm{k}}^{\prime}$ allows us to drop the terms in $n_{\overrightarrow{\mathbf{k}}} n_{\overrightarrow{\mathbf{k}}}$, and $n_{\overrightarrow{\mathbf{k}}+\vec{q}} n_{\overrightarrow{\mathbf{k}}^{\prime}+\vec{q}}$, so that

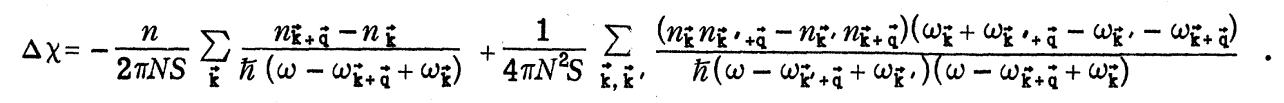

We now use the identity

$$
\left(\omega-\omega_{\overrightarrow{\mathbf{k}}^{\prime}+\overrightarrow{\mathrm{q}}}+\omega_{\overrightarrow{\mathbf{k}}^{\prime}}\right)^{-1}\left(\omega-\omega_{\overrightarrow{\mathbf{k}}+\overrightarrow{\mathrm{q}}}+\omega_{\overrightarrow{\mathbf{k}}}\right)^{-1}=\left[\left(\omega-\omega_{\overrightarrow{\mathbf{k}}+\overrightarrow{\mathrm{q}}}+\omega_{\overrightarrow{\mathbf{k}}}\right)^{-1}-\left(\omega-\omega_{\overrightarrow{\mathbf{k}}^{\prime}+\overrightarrow{\mathrm{q}}}+\omega_{\overrightarrow{\mathbf{k}}^{\prime}}\right)^{-1}\right]\left(\omega_{\overrightarrow{\mathbf{k}}+\overrightarrow{\mathrm{q}}}+\omega_{\overrightarrow{\mathbf{k}}^{\prime}}-\omega_{\overrightarrow{\mathbf{k}}^{\prime}+\overrightarrow{\mathrm{q}}}-\omega_{\overrightarrow{\mathbf{k}}}\right)^{-1},
$$

so that

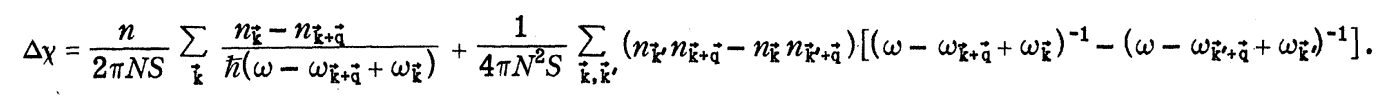

One of the double sums is now trivial, and when it is done, one finds that the double sums exactly cancel the single sum, so that $\Delta \chi=0$.

Thus all three techniques yield the same results to order $1 / S$. Note that in our treatment of the spin operators, it was necessary to treat the discontinuity term accurately in decoupling the spin operators. For this reason our result using spin operators is essentially different from that of $\mathrm{RN}$ and from those of $\mathrm{Liu}^{17}$ and of Reiter. ${ }^{7}$ Our results using the boson formalisms agree (apart from algebraic differences) with those $\mathrm{RN}$ found using the HP formalism.

The equivalence between the various formalisms allows us to make some statements concerning the claims of RN to have detected kinematic effects. They base this claim on the difference between their results for the longitudinal susceptibility using the spin decoupling technique [see their Eq. (12)] and using the HP formalism [see their Eq. (21)]. However, these results cannot be entirely correct, because they do not agree to order $1 / S$, as we have just shown they must. Although we can not prove that the formalisms agree to all orders in $1 / S$, we have every reason to suppose that this is indeed the case. For example, for the transverse dynamical susceptibility the DM transformation (in which the unphysical states are not explicitly excluded from the partition function) does give the same result ${ }^{3}$ as formalisms ${ }^{11,12}$ where such exclusion is systematically carried out. ${ }^{19}$ These results prove that there are no spurious kinematic contributions in the DM formalism from two-spin-wave states, although it is not impossible that such spurious contributions might be introduced by states with many spin waves. In addition, the probability of having $n$ spin deviations on a single site vanishes ${ }^{20}$ (at least to lowest order in $k T / J S)$ within the DM formalism for $n>2 S$, as expected from spin kinematics. Thus, there appears to be a large amount of evidence to show that kinematic interactions are negligible at low temperatures and, consequently, that the DM formalism is a good one.

The situation is much the same for the antiferromagnet. There, the equivalence ${ }^{21}$ (at least to order $1 / S$ ) of the DM and HP transformations (which is only obvious within the physically meaningful states) seems to indicate that these formalisms do not introduce spurious effects from the unphysical states. As in the ferromagnet, the spin-deviation probabilities calculated within the DM formalism preserve the spin kinematics. ${ }^{22}$ The kinematic properties of the dynamical response functions are also correct in that the effects of anisotropy vanish for spin- $\frac{1}{2}$ as they must. ${ }^{23,24}$ Thus, although the possibility of significant kinematic interactions at low temperatures cannot be rigorously excluded, there do not seem to be any well-established results which suggest that such a possibility is realized. 


\section{NONEXISTENCE OF ZERO SOUND AT SHORT WAVELENGTHS}

In order to study a possible zero-sound mode it is necessary to resum the $1 / S$ expansion. In view of the complexities inherent in the spin formalism, it appears that a boson formalism will be simpler. In this connection, it is useful to recall the conclusions reached by Dyson. ${ }^{10}$ He showed rather conclusively that spin waves interact weakly in the long-wavelength limit. Later, Oguchi ${ }^{16}$ showed that the same result could be obtained using the $\mathrm{HP}$ interaction providing the terms were suitably grouped together. The difficulty in the HP formalism is that the bare interaction does not vanish in the longwavelength limit, and therefore the weakness of the interaction is not automatic.

If we solve Eq. (15) by iteration, we obtain a result of the form ${ }^{9}$

$$
\begin{aligned}
H & =H_{0}+H_{0} V H_{0}+H_{0} V H_{0} V H_{0} \cdots \\
& =\left(1-H_{0} V\right)^{-1} H_{0} .
\end{aligned}
$$

For the term $H_{0} V$, RN find near resonance $\left(\omega \approx \omega_{\overrightarrow{\mathrm{q}}}\right)$

$$
H_{0} V \sim \frac{1}{N S} \sum_{\overrightarrow{\mathbf{k}}} \frac{\omega_{\overrightarrow{\mathbf{q}}} n_{\overrightarrow{\mathbf{k}}}}{\omega-\omega_{\overrightarrow{\mathbf{k}}+\vec{q}}+\omega_{\overrightarrow{\mathbf{k}}}},
$$

where the factor $n_{\overrightarrow{\mathbf{k}}}\left(\omega-\omega_{\overrightarrow{\mathbf{k}}+\overrightarrow{\mathrm{q}}}+\omega_{\overrightarrow{\mathbf{k}}}\right)^{-1}$ corresponds to $H_{0}$ in Eq. (25), and $V$ is apparently $\hbar \omega_{\mathrm{q}}$. Since this potential is the interaction between a short-wavelength $(\vec{q})$ magnon and a long-wavelength $(\vec{k})$ magnon, we should expect it to vanish in the limit $k \rightarrow 0$. That it does not is an incorrect result of the formalism used by RN. Parenthetically, one can see that use of a smaller cross section, as is required by our arguments, will make it more difficult to satisfy the condition $1=H_{0} V$, necessary for the existence of a well-defined zero-sound mode.

To take proper account of the weak magnon-magnon interaction we therefore use the DM formalism. Thus, we write the analog of Eq. (15) in the form

$$
\begin{aligned}
\hbar(\omega & \left.-\omega_{\overrightarrow{\mathbf{k}}+\overrightarrow{\mathrm{q}}}+\omega_{\overrightarrow{\mathrm{k}}}\right) H(\overrightarrow{\mathrm{k}}, \overrightarrow{\mathrm{q}} ; \omega)_{\mathrm{DM}} \\
& =\frac{S}{\pi}\left(n_{\overrightarrow{\mathrm{k}}}-n_{\overrightarrow{\mathbf{k}}+\overrightarrow{\mathrm{q}}}\right)+4 \sum_{\overrightarrow{\mathbf{k}}^{\prime}} V_{\overrightarrow{\mathrm{k}}, \overrightarrow{\mathrm{k}}^{\prime}} H\left(\overrightarrow{\mathrm{k}}^{\prime}, \overrightarrow{\mathrm{q}} ; \omega\right)_{\mathrm{DM}} .
\end{aligned}
$$

Since $V_{\overrightarrow{\mathbf{k}} \vec{k}}$, is a sum of separable potentials [see Eq. (19)], we may solve this integral equation in the usual way. We set

$$
H(\overrightarrow{\mathrm{k}}, \overrightarrow{\mathrm{q}} ; \omega)=\frac{\Delta n_{\overrightarrow{\mathbf{k}}}}{\Delta E_{\overrightarrow{\mathbf{k}}}}\left(\frac{S}{\pi}+A_{0}+\sum_{\vec{b}} A_{\vec{b}} e^{i \overrightarrow{\mathbf{k}} \cdot \vec{b}}\right),
$$

where $\Delta n_{\overrightarrow{\mathbf{k}}} \equiv n_{\overrightarrow{\mathbf{k}}}-n_{\overrightarrow{\mathbf{k}}+\overrightarrow{\mathrm{q}}}$ and $\Delta E_{\overrightarrow{\mathbf{k}}} \equiv \hbar\left(\omega-\omega_{\overrightarrow{\mathbf{k}}+\overrightarrow{\mathrm{q}}}+\omega_{\overrightarrow{\mathbf{k}}}\right)$ and the constants $A_{0}$ and $A_{\overrightarrow{6}}$ are determined by substitution into Eq. (27). In this way we find that

$$
A_{0}=\frac{2 J z}{N} \sum_{\overrightarrow{\mathbf{k}}^{\prime}}\left(\gamma_{\overrightarrow{\mathbf{q}}}-\gamma_{\overrightarrow{\mathbf{k}}^{\prime}+\overrightarrow{\mathbf{q}}}\right) \frac{\Delta n_{\overrightarrow{\mathbf{k}}^{\prime}}}{\Delta E_{\overrightarrow{\mathbf{k}}^{\prime}}}\left(\frac{S}{\pi}+A_{0}+\sum_{\vec{b}^{\prime}} A_{\overrightarrow{6}^{\prime}} e^{i \overrightarrow{\mathbf{k}}^{\prime} \cdot \vec{b}^{\prime}}\right),
$$

$$
A_{\vec{b}}=\frac{2 J}{N} \sum_{\overrightarrow{\mathbf{k}}^{\prime}}\left(e^{-i \overrightarrow{\mathrm{k}}^{\prime} \cdot \vec{b}}-1\right) \frac{\Delta n_{\overrightarrow{\mathbf{k}^{\prime}}}}{\Delta E_{\overrightarrow{\mathrm{k}}^{\prime}}}\left(\frac{S}{\pi}+A_{0}+\sum_{\vec{\sigma}^{\prime}} A_{\vec{b}^{\prime}} e^{i \overrightarrow{\mathrm{k}}^{\prime} \cdot \vec{\sigma}^{\prime}}\right),
$$

which enables us to write

$$
A_{0}=-\sum_{\vec{b}} A_{\vec{b}} e^{-i \overrightarrow{\mathrm{a}} \cdot \vec{b}} \text {. }
$$

Using this relation to eliminate $A_{0}$ from Eq. (29b) we obtain

$A_{\vec{b}}=\frac{2 J}{N} \sum_{\overrightarrow{\mathbf{k}}^{\prime}}\left(e^{-i \overrightarrow{\mathbf{k}}^{\prime} \cdot \vec{b}}-1\right) \frac{\Delta n_{\overrightarrow{\mathbf{k}}}}{\Delta E_{\overrightarrow{\mathbf{k}}}}\left(\frac{S}{\pi}+\sum_{\vec{\sigma}^{\prime}} A_{\vec{\sigma}^{\prime}}\left(e^{i \overrightarrow{\mathbf{k}}^{\prime} \cdot \vec{\sigma}^{\prime}}-e^{-i \overrightarrow{\mathrm{a}} \cdot \overrightarrow{\sigma^{\prime}}}\right)\right)$,

which is of the form

$$
A_{\overrightarrow{6}}-\sum_{\vec{\sigma}^{\prime}} K_{\tilde{\sigma}^{\prime}} A_{\overrightarrow{6}^{\prime}}=\frac{2 J}{N} \sum_{\overrightarrow{\mathbf{r}}^{\prime}}\left(e^{-i \overrightarrow{\mathbf{k}}^{\prime} \cdot \vec{\sigma}}-1\right) \frac{S}{\pi} \frac{\Delta n_{\overrightarrow{\mathbf{k}}^{\prime}}}{\Delta E_{\overrightarrow{\mathbf{k}}^{\prime}}},
$$

where

$K_{\overrightarrow{6} \vec{b}^{\prime}}=\frac{2 J}{N} \sum_{\overrightarrow{\mathbf{k}}^{\prime}}\left(e^{-i \overrightarrow{\mathbf{k}^{\prime}} \cdot \vec{\sigma}}-1\right)\left(e^{i \overrightarrow{\mathbf{r}^{\prime} \cdot \vec{b}^{\prime}}}-e^{-i \overrightarrow{\mathrm{a}} \cdot \vec{\sigma}^{\prime}}\right) \frac{\left(n_{\overrightarrow{\mathbf{k}}^{\prime}}-n_{\overrightarrow{\mathbf{k}}^{\prime}+\overrightarrow{\mathbf{q}}^{\prime}}\right)}{\hbar\left(\omega-\omega_{\overrightarrow{\mathbf{k}}^{\prime}+\overrightarrow{\mathbf{q}}}+\omega_{\overrightarrow{\mathbf{k}}^{\prime}}\right)}$.

The regime of interest is $\omega=\omega_{\vec{q}}, \sin \vec{q} \cdot \vec{\delta}=0$ (i. e. , $\vec{q}$ on the Brillouin-zone boundary) because there, as $\mathrm{RN}$ point out, the denominator in Eq. (33) is of order $k^{\prime 2}$. In their case, this leads to a divergence in the $\overrightarrow{\mathrm{k}}^{\prime}$ integral for $\omega=\omega_{\overrightarrow{\mathrm{q}}}$, since

$$
\int \frac{n_{\overrightarrow{\mathbf{k}}^{\prime}}}{\Delta E_{\overrightarrow{\mathbf{k}}^{\prime}}} d \overrightarrow{\mathbf{k}}^{\prime} \sim \int \frac{d k^{\prime}}{\left(\omega-\omega_{\overrightarrow{\mathbf{q}}}\right)+\alpha k^{\prime 2}} \sim\left(\omega-\omega_{\overrightarrow{\mathbf{q}}}\right)^{-1 / 2} .
$$

In our case, the weaker interaction is reflected by the presence of the factor $e^{-i \overrightarrow{\mathbf{k}^{\circ} \cdot \vec{b}}}-1$ which leads to a convergent integral which can be estimated to be of order

$$
K_{\vec{b} \vec{b}} \sim \frac{n}{S} \ll 1 .
$$

(The case $\omega=-\omega_{q}^{*}$ is quite similar, ${ }^{25}$ the possible divergence occurring in that case for $\vec{k}^{\prime} \approx-\vec{q}$.) For completeness we consider briefly the case $\omega=\omega_{\vec{q}}$, for $\vec{q}$ near the Brillouin-zone boundary so that $\nabla_{\vec{q}} \omega=\vec{\epsilon}$, with $\epsilon \ll 1$. Then $K_{\vec{b} \vec{b}^{\prime}}$ can be decomposed into two contributions, one of which is clearly regular and of order $n$, and the other is of the form

$$
I(\epsilon)=\int_{0}^{k} d \overrightarrow{\mathbf{k}}^{\prime} \frac{k^{\prime} \cdot \delta}{\overrightarrow{\mathrm{k}}^{\prime} \cdot \vec{\epsilon}+k^{2}}\left(\frac{k T}{2 J S k^{12}}\right),
$$

where $k_{0}$ is a thermal momentum of order $(k T / J S)^{1 / 2}$. Direct evaluation shows that $I(\epsilon)$ is well behaved for small $\epsilon$, and hence $K_{\overrightarrow{6} \overrightarrow{6}^{\prime}}$ exhibits no anomalous behavior for $\vec{q}$ near, but not on, the Brillouin-zone boundary. The results in Eqs. (35) and (36) show that there is no collective excitation in the longitudinal susceptibility in the short-wavelength regime and that the iterative solution (in powers of $K$ ) to order $1 / S$ is a reasonable approximation at low temperatures. 


\section{CONCLUSION}

We have studied the longitudinal dynamical susceptibility of a Heisenberg ferromagnet at low temperatures in the short-wavelength limit in order to investigate the possible occurrence of zero sound and associated kinematic effects. We find that the weakness of the magnon-magnon interaction precludes the existence of zero sound in this regime and that the longitudinal susceptibility does not appear to show any kinematic effects. Both these conclusions disagree with the recent work of Ranniger and Natoli, ${ }^{9}$ chiefly because of their use of an unphysically strong magnon-magnon interaction and of slight inaccuracies in their decoupling of spin operators.

\section{APPENDIX}

In this Appendix we evaluate the correlation function $\left\langle S_{\overrightarrow{\mathbf{k}}}^{-} S_{-\vec{k}}^{+}\right\rangle$. The calculations are very similar to those given in Ref. 20. We use the DM transformation:

$$
\begin{aligned}
& S^{+}=(2 S)^{1 / 2}\left(1-\frac{a^{\dagger} a}{2 S}\right) a \\
& S^{-}=(2 S)^{1 / 2} a^{\dagger},
\end{aligned}
$$

$$
S^{z}=S-a^{\dagger} a,
$$

where $\left[a, a^{\dagger}\right]=1$. Thus we may write

$$
C_{\overrightarrow{\mathbf{k}}} \equiv\left\langle S_{\overrightarrow{\mathbf{k}}}^{-} S_{-\overrightarrow{\mathbf{k}}}^{+}\right\rangle=2 S\left\langle a_{\overrightarrow{\mathbf{k}}}^{\dagger} a_{\overrightarrow{\mathbf{k}}}\right\rangle-N^{-1} \sum_{\overrightarrow{\mathfrak{j}} \overrightarrow{\mathrm{q}} \overrightarrow{\mathbf{r}}}\left\langle a_{\overrightarrow{\mathbf{k}}}^{\dagger} a_{\overrightarrow{\mathfrak{p}}}^{\dagger} a_{\overrightarrow{\mathbf{q}}} a_{\overrightarrow{\mathbf{r}}}\right\rangle .
$$

The four-boson correlation function can be evaluated diagrammatically by a slight modification of the rules given by Bloch and de Dominicis ${ }^{26}$ for the free energy. One simply evaluates free-energy diagrams with an extra vertex representing the correlation function which is to be evaluated. ${ }^{20}$ For this evaluation we write the Hamiltonian as

$$
\mathfrak{H C}=\sum_{\overrightarrow{\mathrm{k}}} \hbar \omega_{\overrightarrow{\mathbf{k}}} a_{\overrightarrow{\mathrm{k}}}^{\dagger} a_{\overrightarrow{\mathrm{k}}}+\sum_{1234} V_{12 ; 34} a_{k_{1}}^{\dagger} a_{k_{2}}^{\dagger} a_{k_{3}} a_{k_{4}},
$$

where

$V_{12 ; 34}=(J z / 2 N)\left(\gamma_{1}+\gamma_{2}-\gamma_{1-3}-\gamma_{1-4}\right) \delta\left(\overrightarrow{\mathbf{k}}_{1}+\overrightarrow{\mathbf{k}}_{2}-\overrightarrow{\mathbf{k}}_{3}-\overrightarrow{\mathbf{k}}_{4}\right)$

is the DM interaction written in slightly different form than in Eq. (19). Here $\gamma_{1} \equiv \gamma_{\vec{k}_{1}}$, etc.

Following the treatment in Ref. 20, we find that

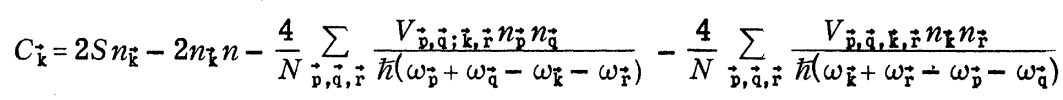

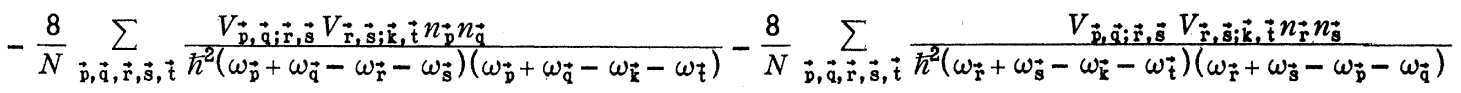

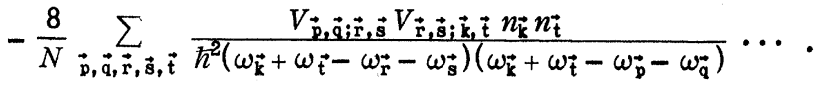

This series includes all terms with two occupation numbers. The corresponding diagrams are the familiar ladder graphs shown in Ref. 20. It is clear that we may obtain a lowest-order evaluation by setting to zero those momenta which appear in occupation numbers. Note that the DM interaction vanishes when either of its last two subscripts vanishes. Thus, most of the terms in the series may be dropped and we find that

$$
\begin{aligned}
& C_{\overrightarrow{\mathrm{k}}}=2 S n_{\overrightarrow{\mathrm{k}}}-2 n n_{\overrightarrow{\mathrm{k}}}+\frac{4}{N} \sum_{\overrightarrow{\mathfrak{j}}, \overrightarrow{\mathrm{q}}, \overrightarrow{\mathrm{r}}} \frac{V_{\overrightarrow{\mathrm{p}}, \overrightarrow{\mathrm{q}} ; \overrightarrow{\mathrm{k}}, \overrightarrow{\mathrm{r}}} n_{\overrightarrow{\mathrm{j}}} n_{\overrightarrow{\mathrm{g}}}\left(\omega_{\overrightarrow{\mathrm{k}}}+\omega_{\overrightarrow{\mathrm{r}}}\right)}{\omega^{\prime}}
\end{aligned}
$$

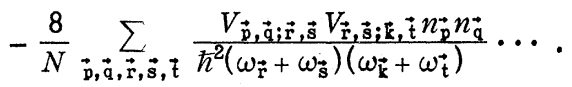

Note also that

$$
\left.V_{12 ; 34}\right|_{k_{1}=k_{2}=0}=(\hbar / 4 S N)\left(\omega_{3}+\omega_{4}\right) \delta\left(\overrightarrow{\mathrm{k}}_{3}+\overrightarrow{\mathrm{k}}_{4}\right),
$$

so that

$\frac{4}{N} \sum_{\overrightarrow{\mathfrak{p}}, \vec{q}, \overrightarrow{\mathrm{r}}} \frac{V_{\overrightarrow{\mathrm{p}}, \overrightarrow{\mathrm{q}}, \overrightarrow{\mathrm{k}}, \overrightarrow{\mathrm{r}}} n_{\overrightarrow{\mathrm{p}}} n_{\overrightarrow{\mathrm{q}}}}{\hbar\left(\omega_{\overrightarrow{\mathrm{k}}}+\omega_{\overrightarrow{\mathrm{r}}}\right)}=\frac{n^{2}}{S}$,

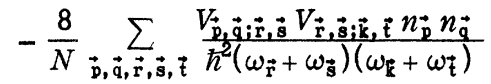

$$
=-\frac{2 n^{2}}{S} \sum_{\overrightarrow{\mathbf{r}}} \frac{V_{\vec{r}_{1}-\overrightarrow{\mathbf{r}}_{j} ; \overrightarrow{\mathbf{k}}_{2}-\overrightarrow{\mathbf{k}}}}{2 \hbar \omega_{\overrightarrow{\mathbf{k}}}}=0 .
$$

Thus the terms in Eq. (A5) yield

$$
C_{\overrightarrow{\mathbf{k}}}=2 S n_{\overrightarrow{\mathbf{k}}}-2 n n_{\overrightarrow{\mathbf{k}}}+n^{2} / S .
$$

Higher-order terms in Eq. (A5) can be shown to vanish using the arguments of Ref. 20.

This result is consistent with the result ${ }^{27}$ obtained by Wortis for $\left\langle S_{\vec{R}}^{-} S_{\vec{R}}^{+}\right\rangle$, since

$$
\begin{aligned}
N^{-1} \sum_{\overrightarrow{\mathbf{R}}}\left\langle S_{\overrightarrow{\mathbf{k}}}^{-} S_{-\overrightarrow{\mathbf{k}}}^{+}\right\rangle & =\left\langle S_{\overrightarrow{\mathrm{R}}}^{-} S_{\overrightarrow{\mathrm{R}}}^{+}\right\rangle \\
& =2 S n-2 n^{2}[1-(1 / 2 S)] .
\end{aligned}
$$


*Work supported in part by the Office of Naval Research and the Advanced Research Projects Agency.

${ }^{1}$ M. Wortis, Phys. Rev. 132, 85 (1963).

${ }^{2}$ R. G. Boyd and J. Callaway, Phys. Rev. 138, A1621 (1965).

${ }^{3}$ R. Silberglitt and A. B. Harris, Phys. Rev. 174, 640 (1968).

${ }^{4}$ R. J. Elliott and M. F. Thorpe, J. Phys. C 2 , 1630 (1969).

${ }^{5}$ R. B. Dingle, Proc. Phys. Soc. (London) $\underline{\mathrm{A} 65}, 1044$ (1952).

${ }^{6}$ R. N. Gurzhi, Fiz. Tverd. Tela $\underline{7}, 3515$ (1965) [Sov. Phys. Solid State 7, 2838 (1966)].

${ }^{7}$ G. Reiter, Phys. Rev. 175,631 (1968)。

${ }^{8}$ T. Izuyama and M. Saitoh, Phys. Letters $\underline{29 \mathrm{~A}}, 581$ (1969).

${ }^{9} \mathrm{~J}$. Ranninger and C. R. Natoli (unpublished), hereafter referred to as $\mathrm{RN}$.

${ }^{10}$ F. J. Dyson, Phys. Rev. $\underline{102}, 1217$, (1956); $\underline{102}$, 1230 (1956).

${ }^{11} \mathrm{~J}$. F. Cooke and H. H. Hahn, Phys. Rev. B 1,1243 (1970).

${ }^{12}$ J. I. Davis, Ann。 Phys. (N。Y.) 58, 529 (1970), see especially Eqs. (6.9) and (6.12).

${ }^{13}$ A. B. Harris, Phys. Rev. 175, 674 (1968)。

${ }^{14}$ S. V. Maleev, Zh. Eksperim。 i Teor。Fiz. $\underline{33}, 1010$ (1957) [Sov. Phys. JETP $\underline{6}, 776$ (1956)].
${ }^{15}$ T. Holstein and H. Primakoff, Phys. Rev. $\underline{58}, 1098$ (1940).

${ }^{16} \mathrm{~T}$. Oguchi, Phys. Rev. 117, 117 (1960).

${ }^{17}$ S. H. Liu, Phys. Rev. 139, A1522 (1965).

${ }^{18}$ D. N. Zubarev, Usp. Fiz。 Nauk 71, 71 (1960) [Sov. Phys. Usp. $\underline{3}, 320$ (1960)].

${ }^{19} \mathrm{R}$. Silberglitt and A。B. Harris, Comments Solid State Phys. $\underline{3}, 8$ (1970).

${ }^{20}$ A. B. Harris, J. Phys. Chem. Solids 28,1579 (1967).

${ }^{21}$ A. B. Harris, J. Phys. Chem. Solids 27,1927 (1966).

${ }^{22}$ A. B. Harris, Phys. Rev. Letters 21, 602 (1968).

${ }^{23}$ A. B. Harris, Phys. Rev. 183, 486 (1969).

${ }^{24}$ A. B. Harris, D. Kumar, B. I. Halperin, and P、 C. Hohenberg, Phys. Rev. B $\underline{3}, 961$ (1971).

${ }^{25}$ From the definition of $\chi_{\vec{q}}^{z z}(\omega)$, we have that $\chi_{\vec{q}}^{z z}(\omega)$ $=\chi_{q}^{z z}(-\omega)$. Since $V_{\mathrm{DM}}$ is not Hermitian, it does not automatically preserve this property. However, if Eq. (27) is solved by iteration, one can show that to each order in $V_{\mathrm{DM}}, \chi_{q}^{z}(\omega)$ is an even function of $\omega$, although the more stringent condition $H(\vec{k}, \vec{q} ; \omega)=H(-\vec{k}-\vec{q}, \vec{q} ;-\omega)$ is not preserved by $V_{\mathrm{DM}}$. We would like to thank Dr. Ranninger for bringing this question to our attention.

${ }^{26} \mathrm{C}$. Bloch and C. de Dominicis, Nucl. Phys. 7,459 (1958).

${ }^{27} \mathrm{M}$. Wortis, Ph. D. thesis (Harvard University, 1963) (unpublished). 